\title{
Diseño y validación de una escala observacional sobre el estilo motivador
} docente

\section{Design and validation of a scale for measuring motivational teaching style}

\section{Desenho e validação de uma escala observacional sobre o estilo de ensino motivador}

\author{
Barrachina-Peris, J. ${ }^{1}$, Moreno-Murcia, J. A. ${ }^{2}$ y Huéscar, E. ${ }^{3}$ \\ ${ }^{1}$ IES Mediterranea, Benidorm, Generalitat Valenciana (España), ${ }^{2}$ Centro de Investigación del Deporte, \\ Universidad Miguel Hernández de Elche (España).,; Departamento de Psicología de la Salud, \\ Universidad Miguel Hernández de Elche (España)
}

\section{RESUMEN}

La metodología observacional permite analizar el estilo motivacional que despliega el docente en clase y proporciona una nueva perspectiva para interpretar la influencia de las interacciones docentes en el proceso de instrucción. El objetivo de este trabajo fue diseñar una escala de estimación ad hoc para la Medida del Estilo Interpersonal Docente (MEID) y validarla Participaron 30 docentes universitarios (16 mujeres y 14 hombres) con una edad media de 46.1 años $(D T=5.8)$ y con una experiencia docente de 15.71 años $(D T=6.9)$. La calidad del dato fue alcanzada a través de la técnica de validez de contenido y el aspecto cuantitativo fue tratado por medio del análisis del W de Kendall, ajustando un modelo final de 60 ítems agrupados en 4 dimensiones exhaustivas y mutuamente excluyentes: apoyo a la autonomía, apoyo a la estructura antes de la tarea, apoyo a la estructura durante la tarea y apoyo a la relación. Las evidencias iniciales de la escala MEID lo presentan como un instrumento psicométrico robusto y fiable para poder ser empleado como técnica observacional del estilo interpersonal docente. Los resultados obtenidos abren nuevas líneas de estudio en las que la escala MEID podría ser empleada en programas formativos encaminados a la optimización de patrones motivacionales docentes adaptativos.

Palabras clave: Necesidades Psicológicas Básicas; Teoría de la Autodeterminación; Enseñanza; Observación; Evaluación.

\begin{abstract}
The observational methodology allows the analysis of the motivational style displayed by the teacher in class and provides a new perspective to interpret the influence of teacher interactions in the instructional process. The aim of this work was to design an ad hoc estimation scale for the Measure of Interpersonal Teaching Style (MEID) and to validate it. 30 university teachers (16 women and 14 men) with a mean age of 46.1 years $(S D=5.8)$ and with a teaching experience of 15.71 years $(S D=6.9)$ participated. Data quality was achieved through the content validity technique by expert judgment and the quantitative aspect was treated through Kendall's W analysis, fitting a final model of 60 items grouped into 4 exhaustive and mutually exclusive dimensions: autonomy support, pre-task structure support, on-task structure support, and relationship support. The initial evidence of the MEID scale presents it as a robust and reliable psychometric instrument to be used as an observational technique of interpersonal teaching style. The results obtained open new lines of study in which the MEID scale could be used in training programs aimed at optimizing adaptive motivational teaching patterns.
\end{abstract}

Keywords: Basic Psychological Needs, Self-Determination Theory, Teaching, Observation, Evaluation. 


\section{Barrachina-Peris et al.}

\section{RESUMO}

A metodologia observacional permite-nos analisar o estilo motivacional que os professores exibem na aula e fornece uma nova perspectiva para interpretar a influência das interacções dos professores no processo instrucional. O objectivo deste trabalho era conceber uma escala de estimativa ad hoc para a Medida de Estilo de Ensino Interpessoal (MEID) e validá-la. Trinta professores universitários (16 mulheres e 14 homens) com uma idade média de 46,1 anos $(S D=5,8)$ e com uma experiência de ensino de 15,71 anos $(S D=6,9)$ participaram no estudo. A qualidade dos dados foi conseguida através da técnica de validade do conteúdo através do julgamento de peritos e o aspecto quantitativo foi tratado através da análise $\mathrm{W}$ de Kendall, encaixando um modelo final de 60 itens agrupados em 4 dimensões exaustivas e mutuamente exclusivas: suporte de autonomia, suporte de estrutura pré-tarefa, suporte de estrutura na tarefa, e suporte de relacionamento. A evidência inicial da escala MEID apresenta-a como um instrumento psicométrico robusto e fiável para ser utilizado como uma técnica de observação do estilo de ensino interpessoal. Os resultados obtidos abrem novas linhas de estudo nas quais a escala MEID poderia ser utilizada em programas de formação destinados a optimizar os padrões motivacionais adaptativos nos professores

Palavras chave: Necessidades Psicológicas Básicas; Teoria da auto-determinação; Ensino; Observação; Avaliação.

\section{INTRODUCCIÓN}

La Teoría de la Autodeterminación (TAD, Ryan y Deci, 2000) viene siendo un marco teórico de referencia para explicar la orientación motivacional que subyace al compromiso, la persistencia y a la alienación en las actividades académicas, así como la catalización que ejercen los factores sociales, entre los que se encuentra el docente. A partir de la satisfacción o frustración de las necesidades psicológicas básicas, la interacción del docente en clase con sus estudiantes se presenta como una variable clave por sus efectos sobre la calidad de la motivación de éstos, y consecuentemente, sobre una serie de desencadenantes finales de diferente naturaleza (cognitiva, emocional o comportamental) (Reeve y Tseng, 2011; Skinner, Kindermann, Connell, y Wellborn, 2009; Wallace, Kelcey, y Ruzek, 2016).

En la última década emerge una línea de estudio del estilo interpersonal motivador que despliega el docente en clase por medio de la observación, orientada a mejorar la calidad de la enseñanza y promover la motivación autodeterminada en los estudiantes (Aelterman et al., 2018; Abós, Sevil, Martín-Albo, y García-González, 2018; Moreno-Murcia, 2016).

La metodología observacional se erige como una metodología científica, cuya estructura flexible hace posible adaptar los diseños observacionales a la realidad perceptible y permite recoger información directamente de los participantes en su contexto natural, sin necesidad de elicitar la respuesta (Anguera y Hernández-Mendo, 2013, 2014). Mediante la construcción de instrumentos de observación elaborados ad hoc, se puede obtener un sistema de categorías exhaustivo y de exclusión mutua (Anguera y Hernández-Mendo, 2014; Anguera, Blanco, HernándezMendo y Losada, 2011; Anguera, Blanco, Losada y
Hernández-Mendo, 2000). La metodología observacional está completamente asentada en el contexto deportivo y puede considerarse en sí misma como mixed method, atendiendo a combinación de datos cualitativos y cuantitativos o por combinarse con otras metodologías diferentes a la observacional dentro de una estructura de investigación más amplia (Anguera, Camerino, Castañer y Sánchez-Algarra, 2014; Anguera y Hernández-Mendo, 2016; Anguera, Camerino, Castañer, Portell, y SánchezAlgarra, 2020).

Enmarcado en la metodología observacional, para diseñar y validar la Escala de Medida Observacional del Estilo Interpersonal Docente (MEID), el presente estudio se elaboró a partir de la adaptación de las etapas propuestas por Anguera y Hernández-Mendo (2013): delimitación del problema y propuesta de diseño observacional (diseño y validación de una escala para medir la interacción docente empleando una metodología mixta) recogida, gestión y optimización de datos (mediante autoinformes y preguntas abiertas) análisis de datos e interpretación (W Kendall y análisis de narrativas/textos) con el objetivo de obtener un diseño y validar la escala posteriormente.

\section{Estilo interpersonal motivador}

Reeve (2009) define al estilo interpersonal como las prácticas adoptadas por los docentes para estimular el proceso de aprendizaje, la motivación y el desarrollo personal de los estudiantes. Un estilo motivador altamente estructurado y con gran apoyo a la autonomía de los estudiantes genera un clima en clase que incrementa la motivación intrínseca (Davis, 2018; Jang et al., 2010; Moreno-Murcia, y Sánchez-Latorre, 2016), mejora el compromiso (Meng, Wang, y Keng, 2016, Reeve Jang, Carrell, Jeon, y Barch, 2004), el aprendizaje profundo (Cheon, Reeve y Moon, 2012; Nuñez y León, 2016; Jang, 


\section{Escala observacional del estilo motivador en la educación física}

Reeve, y Halusic, 2016; Jang. Reeve, Ryan, y Kim, 2009), los resultados académicos (Griffin, 2016; Gunuc, 2014), el bienestar (Assor, Kaplan, y Roth, 2002) y disminuye la desmotivación (Cheon y Reeve, 2015), mientras que un estilo altamente controlador, se asocia a resultados negativos (Haerens, Vansteenkiste, Aelterman, y Van den Berghe, 2016; Jang, Reeve, y Deci, 2010).

\section{Medición del estilo motivacional docente}

El estudio del estilo motivacional docente se ha abordado mayormente desde dos enfoques: experimental (Cheon, Reeve, y Ntoumanis, 2018; Cheon, Reeve, y Song, 2016) y autoinformes (Haerens et al., 2013). En el experimental, se aíslan dimensiones y se comparan con la variante experimental, quedando limitada su validez ecológica (Haerens et al., 2013). Los autoinformes, miden las percepciones de docentes y discentes sobre las prácticas y ambientes para dar apoyo a la autonomía, permitiendo comprenderlas con mayor naturalidad y facilitando estrategias para implementarlas (Haerens et al., 2013; Reeve, 2009; Reeve y Jang, 2006; Jang et al., 2016). Por medio de autoinformes se ha analizado las estrategias para dar apoyo a la autonomía (Cheon et al., 2014), su persistencia en el tiempo (Cheon y Reeve, 2013), el apoyo a la autonomía como un factor único (Soenens y Vansteenkiste, 2005; Zhan, Solmon, Kosma, Carson, y Gu, 2011) y el apoyo a la autonomía y la estructura conjuntamente (Jang, Reeve, y Halusic, 2016).

Entre sus limitaciones se encuentran la existencia de discrepancias entre la percepción del proceso y la realidad (Aguado-Gómez, Díaz-Cueto, Hernández-Álvarez, y López-Rodríguez, 2016; León et al., 2018) o la dificultad para interpretar las conductas docentes en el contexto en que son desplegadas y el impacto real que éstas producen en el estudiante (Jiang, Vauras, Volet, Salo, y Kajamies, 2019).

En el contexto educativo, estudio observacional se está consolidando como una línea de investigación orientada al microanálisis de las conductas que el docente despliega al aplicar el estilo motivacional de apoyo a la autonomía, que complementa a los enfoques anteriores (Rouse, Duda, Ntoumanis, Jolly, y Williams, 2016; Smith et al., 2015). Haerens et al. (2013) sostienen que el estudio observacional hará posible validar las prácticas empleadas para dar apoyo a la autonomía, permitiendo diseñar un sistema de códigos que defina cuándo se proporcionan unas estrategias u otras, mejorando la fiabilidad entre la objetividad de las conductas docentes implementadas y la subjetividad percibida con el autoinforme y ayudará a validar un mapa observacional que permita evaluar el impacto en futuras intervenciones, de manera que facilite la formación del docente en el estilo de apoyo a la autonomía. El estudio observacional permitió a Jiang et al. (2019) demostrar la relación de interdependencia entre el contexto y el empleo de estrategias novedosas para dar apoyo a la autonomía, como la tolerancia al error o la enseñanza creativa, así como identificar comportamientos de control indirecto, como avergonzar a los estudiantes en público o combinar un clima ego con explicaciones razonadas, que no se apreciaban con autoinformes.

Estos hallazgos indican que la metodología observacional puede convertirse en una metodología idónea para ahondar en la comprensión del estilo motivacional docente, puesto que renueva las perspectivas de análisis del conocimiento científico e impulsa la elaboración de diseños mixtos (mixed-methods), aportando nuevos instrumentos de medida y permitiendo avanzar en las propias técnicas de análisis de los datos, como viene realizándose hace décadas en el contexto deportivo (Anguera et al., 2018; Anguera y Hernández-Mendo, 2014, 2016) . No obstante, hasta la fecha son escasos los instrumentos desarrollados para medir el estilo interpersonal docente en el seno de la metodología observacional, apreciándose limitaciones en el procedimiento para la medición de las conductas, así como en las relaciones de causalidad entre las dimensiones y los comportamientos. Reeve y Jang (2006) desarrollaron un instrumento que confrontaba el estilo controlador y el apoyo a la autonomía a través de 21 comportamientos. Las limitaciones se concentran en la naturaleza de las conductas a medir (tiempo que el docente monopoliza el material, tiempo que el docente está escuchando a los estudiantes, tiempo que deja que trabajen autónomamente, etc.) y en el procedimiento para la medida (recuento de segundos empleados en cada acción y frecuencia con las que se repiten). Tessier, Sarrazin y Ntoumanis (2008) diseñaron un instrumento que comparaba el estilo controlador y apoyo a la autonomía e incorporaba el estilo neutro. Estructurado en ocho categorías de análisis, acotaba el registro a la parte central de la clase (20-30 minutos). Las limitaciones se asocian con los comportamientos a observar (frecuencia con la que los aspectos organizativos se imponen, frecuencia con que se dirige a los estudiantes empleando una entonación controladora, etc.) y en las dimensiones planteadas (organización de la clase, instrucciones técnicotácticas, preguntas, elogios, apoyos, control de la disciplina y críticas), que podían dar lugar a interpretaciones ambiguas. Haerens et al. (2013) propusieron un instrumento para medir el apoyo a la autonomía que contemplaba 21 conductas, agrupadas en cuatro factores (apoyo a la autonomía, estructura antes de la práctica, estructura durante la práctica y apoyo a la relación). Las limitaciones giran en torno al procedimiento para medir (registro de las conductas en intervalos de 5 minutos), en la delimitación y ambigüedad de ciertos comportamientos (pone esfuerzo y energía dando la clase) y en la ausencia de medida del estilo controlador, que podría dar lugar a una interpretación del estilo motivacional docente sesgada.

En el seno de la metodología observacional el objetivo del estudio es el de desarrollar una escala de estimación (o lista de control) para el desarrollo del estilo motivacional 


\section{Barrachina-Peris et al.}

docente, y aportar evidencias de su fiabilidad y validez. Se pretende mejorar la efectividad de la enseñanza por medio del empleo de una escala observacional que toma como unidad de medida la tarea. Concentrar el análisis en las tareas y no en intervalos temporales dentro de una unidad observacional pone el acento en la calidad de la instrucción y en el clima que genera el docente en clase, de acuerdo con los estudios observacionales desarrollados hasta la fecha en el ámbito de la enseñanza (Smith, Quested, Appleton, y Duda, 2016; Jiang et al., 2019). Smith et al. (2016) y Jiang et al. (2019) cuestionan el empleo de la frecuencia como indicador válido para mejorar la calidad de los estudios observacionales, a pesar de ser una medida habitual y proponen analizar el ambiente (contexto) y el mensaje proporcionado por el docente, como indicadores más apropiados para medir la calidad de la instrucción.

Por tanto, se espera que definir con mayor suficiencia, coherencia, relevancia y claridad las conductas que el docente emplea cuando propone y desarrolla las tareas, otorgue mayor calidad al dato, permita analizar con más profundidad qué estilo motivacional está poniendo en práctica (controlador, neutro o apoyo a la autonomía), ayude a delimitar las estrategias que emplea de forma más precisa y sirva para minimizar las limitaciones de los instrumentos observacionales actuales.

\section{MATERIAL Y MÉTODOS}

\section{Diseño observacional}

Se siguió un diseño observacional nomotético, de seguimiento inter sesional y multidimensional (Anguera et al., 2011; Anguera y Hernández-Mendo, 2013; HernándezMendo, 2000) atendiendo a los participantes, al seguimiento y a las diversas dimensiones en las cuales se despliegan los criterios y categorías elaboradas ad hoc para estos estudios.

\section{Participantes}

Se obtuvieron los permisos legales necesarios del órgano evaluador de proyectos de la institución del investigador principal (DPS.JMM.01.14).

Siguiendo el método Delphi (Reguant-Álvarez y TorradoFonseca, 2016), para validar el instrumento se conformaron dos grupos: coordinador y expertos. El grupo coordinador estuvo integrado por tres expertos en el análisis de concordancia de $\mathrm{W}$ de Kendall que atesoraban una dilatada trayectoria en el campo de la investigación colaborativa. Participó activamente en el proceso de diseño y validación de la escala. El grupo de expertos se compuso de 30 docentes universitarios (16 mujeres y 14 hombres) con edades comprendidas entre los 36 y 56 años $(M=46.1, D T$ $=5.8)$, con una amplia experiencia docente $(M=15.71$ años; $D T=6.9$ ) relacionada con la evaluación de instrumentos de esta naturaleza (Escobar-Pérez y Cuervo-Martínez, 2008).
En la segunda fase, participaron 10 docentes universitarios (4 mujeres y 6 hombres) con edades comprendidas entre 26 y 53 años $(M=38.3$ años; $D T=6.3)$. Los objetivos en ambas fases se centraron en el diseño ad hoc de una escala de estimación para registrar la interacción docente y se alcanzó la concordancia consensuada.

\section{Instrumentos}

Estilo Motivador Docente. Se diseñó la escala de estimación para la Medida del Estilo Interpersonal Motivador Docente (MEID) que quedó compuesta por 60 ítems, precedidos por el enunciado "durante la instrucción el docente" y agrupados en cuatro dimensiones: apoyo a la autonomía, con cinco ítems para el apoyo a la autonomía (e.g., "Pregunta al estudiante sobre sus preferencias en relación a una tarea") y cinco para el estilo controlador (e.g., "No pregunta ni tiene en cuenta las preferencias del estudiante"), apoyo a la estructura antes de la tarea, con cinco ítems para el apoyo a la autonomía (e.g., "Al iniciar la clase explica y razona los objetivos") y cinco para el estilo controlador (e.g., "No explica los objetivos y se centra en el contenido"), apoyo a la estructura durante la tarea, con ocho ítems para apoyo a la autonomía (e.g. "Adapta las instrucciones según el progreso de los estudiantes"), ocho para estilo controlador (e.g. "Mantiene constantes las informaciones, con independencia del progreso (empleando un lenguaje controlador") y cuatro para estilo neutro (e.g., "Proporciona informaciones sin promover el progreso ni emplear un lenguaje controlador") y apoyo a la relación, con siete ítems para apoyo a la autonomía (e.g., "Emplea un lenguaje empático"), siete para estilo controlador (e.g., "No emplea un lenguaje empático y no se adapta a los estudiantes") y cinco para estilo neutro (e.g., "Utiliza un lenguaje apático cuando se dirige a los estudiantes, sin emoción, no aporta nada, ni positivo ni negativo)".

\section{Procedimiento}

Obtenidos los permisos pertinentes, seguidamente se configuró el grupo coordinador y se diseñó la estructura general para el proceso de diseño y validación del instrumento. La secuencia completa de las fases para la construcción de la escala MEID quedó compuesta por las tres etapas que presenta el cronograma (Figura 1):

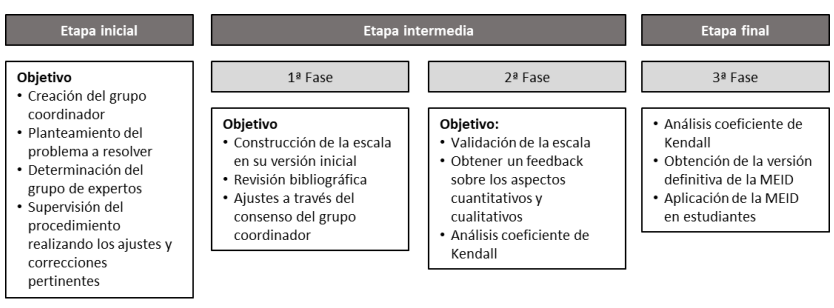

Figura 1. Etapas para el diseño de la escala MEID 


\section{Escala observacional del estilo motivador en la educación física}

En una etapa inicial se constituyó el grupo coordinador, se planteó el problema de investigación y se formuló la hipótesis de partida. Se acotó el diseño de investigación y se establecieron las fases generales para elaborar y validar el instrumento. Seguidamente se pasó al diseño del instrumento. Para ello se llevó a cabo una exhaustiva revisión bibliográfica, acotando el intervalo de análisis añoaño. Se consultaron las siguientes bases de datos: Science Direct, Sport Discus, Scopus, Psycoinfo, Isi web of Knowledge, Medline y TPSR Alliance. A partir de los instrumentos existentes, (Haerens et al., 2013; Reeve, 2009; Reeve et al., 2014; Reeve y Jang, 2006; Tessier et al., 2008), se adaptaron los reactivos o ítems y se diseñó una escala para medir el estilo motivador docente de apoyo a la autonomía frente al estilo controlador y neutro. La escala fue ajustada por los coordinadores empleando la técnica Delphi y se analizó su consistencia interna. A continuación, se procedió con la validez de contenido.

Para medir la validez del instrumento se puntuó cada variable o ítem empleando una escala Likert, ordenada desde 1 (no cumple con el objetivo) hasta 4 (alto nivel de cumplimiento), que puntuó la suficiencia (los ítems que pertenecen a una misma dimensión bastan para obtener la medición de ésta), la claridad (el ítem se comprende fácilmente, posee una sintáctica y semántica adecuadas), la coherencia (el ítem tiene relación lógica con la dimensión que está midiendo) y la relevancia (el ítem es esencial o importante). Además, cada ítem iba acompañado de una pregunta abierta para recoger cualquier valoración cualitativa. Con la intención de lograr evidencias que apoyaran la validez conceptual, cultural y lingüística de la escala, se recopilaron los datos para el análisis de contenido empelando la técnica de juicio de expertos y se calculó el W de Kendall.

Posteriormente se calculó la validez de comprensión del instrumento de observación y se realizó un primer estudio piloto. Una vez recopiladas las puntuaciones, el grupo coordinador evaluó la información obtenida en la escala Delphi desde el punto de vista cuantitativo (escala Likert) y cualitativo (pregunta abierta). Se midió el grado de consenso de todas las respuestas utilizando el W de Kendall y el análisis del discurso obtenido con la pregunta abierta. Para sintetizar, organizar y categorizar la información del ítem abierto, se crearon unidades de análisis de significado en torno a metacódigos (dimensiones del constructo) y códigos (ítems) vinculados a la suficiencia, claridad, relevancia y coherencia. La categorización se realizó cumpliendo los requisitos de exhaustividad, exclusión mutua y un único principio clasificatorio. Con los datos obtenidos, se modificaron algunos ítems y se envió la escala a un número reducido de expertos con ejemplos aclaratorios en los ítems que obtuvieron una baja puntuación en la revisión anterior. Se analizó nuevamente el grado de consenso y se obtuvieron valores adecuados quedando definida la escala MEID. Para comprobar la validez de comprensión, se envió la escala a una muestra independiente compuesta por 10 docentes universitarios que confirmaron los resultados anteriores.

Finalmente, para comprobar la validez y fiabilidad y estimar la generalizabilidad de la escala MEID, se llevó a cabo un estudio piloto para dar apoyo a la autonomía. Se registraron en vídeo 8 clases y se comprobó la fiabilidad inter e intra observador. Para validar la fiabilidad intra observador, se midió la misma clase en dos ocasiones, separando ambas medidas por un intervalo temporal de 15 días. La fiabilidad inter observador fue lograda poniendo en común las medidas obtenidas y consensuando las interpretaciones cuando fue necesario. El grupo coordinador supervisó todo el proceso de validación de la fiabilidad y validez de la escala.

\section{Análisis estadístico}

Los datos cualitativos han sido analizados mediante análisis de contenido. El análisis de los datos cuantitativos se ha realizado con el programa SPSS 25.0.

La construcción de la escala de estimación se elaboró ad hoc a partir de los modelos teóricos existentes y la adaptación a la realidad (Anguera y Hernández-Mendo, 2013, 2014) que presentaba unas unidades de observación (conductas que despliega el docente en clase acotados en los ítems) cuyo registro se realizó de manera continuada a lo largo de cada sesión. Atendiendo al objetivo del estudio, se emplearon parámetros primarios de registro en torno a la frecuencia. Para garantizar la exhaustividad, de acuerdo con Anguera y Hernández-Mendo (2013), previamente se aplicó una prueba de cautela de tres sesiones en las que se comprobó que en el desarrollo del proceso de instrucción no concurrían nuevas unidades de observación distintas a las listadas en el instrumento y se comprobó la mutua exclusividad del sistema de categorías. La consistencia intersesional persigue garantizar la máxima homogeneidad entre las diversas sesiones (Hernández-Mendo, 2000) y se verificó en el proceso de validación de contenido cuando el grupo de expertos utilizó la escala MEID para analizar diferentes situaciones de enseñanza-aprendizaje filmadas en vídeo.

\section{RESULTADOS}

Primera fase. Revisión bibliográfica. Tras el planteamiento de la hipótesis y el establecimiento del diseño a seguir, se estableció una primera versión de la escala organizada en 4 dimensiones y 25 unidades de observación. A través de la técnica Delphi la escala quedó definida por 60 ítems y cuatro dimensiones: apoyo a la autonomía, apoyo a la estructura antes de la tarea, apoyo a la estructura durante la tarea y apoyo a la relación, con una adecuada consistencia interna. Obtenidos los índices de fiabilidad, se estableció la validez de contenido (Anguera, 1990; Anguera y 


\section{Barrachina-Peris et al.}

Hernández-Mendo, 2013). Los análisis proporcionaron evidencias que apoyaron la validez conceptual, cultural y lingüística de la escala. En general se obtuvieron valores adecuados para el W de Kendall, excepto en el factor claridad que arrojó valores discretos en algunos ítems (Tabla 1).

La revisión de los instrumentos existentes culminó con la primera versión de la escala que contó con 25 ítems, precedidos por el encabezado "Cuando el docente está dando clase" y agrupada en un constructo formado por cuatro dimensiones: autonomía, con siete ítems (e.g., "Frecuencia con la que deja tomar la iniciativa a los estudiantes"), estructura antes de la enseñanza, con cuatro ítems (e.g., "Frecuencia con la que expone la estructura de la sesión"), estructura durante la enseñanza, con siete ítems (e.g., "Frecuencia con la que proporciona refuerzos positivos") y relación, con siete ítems (e.g., "Frecuencia con la que atiende las dudas y problemas de los estudiantes"). A través de la técnica Delphi la escala quedó definida por 60 ítems y cuatro dimensiones: apoyo a la autonomía, apoyo a la estructura antes de la tarea, apoyo a la estructura durante la tarea y apoyo a la relación con una adecuada consistencia interna. Para la interpretación de dichas dimensiones, se utilizó la tarea como unidad significante mínima de la instrucción del docente en clase, representando el indicador de referencia para la utilización de la escala. Por lo tanto, la estructura del registro final consistiría en la interpretación dentro de la tarea de las cuatro dimensiones de análisis propuestas anteriormente, dando lugar a una matriz individual compuesta por frecuencias de conductas para cada dimensión.

Segunda fase. Validez de contenido del instrumento. Mediante la técnica de juicio de expertos se obtuvieron evidencias que apoyaron la validez conceptual, cultural y lingüística de la escala. En general se obtuvieron valores adecuados para el W de Kendall, excepto en el factor claridad que arrojó valores discretos en algunos ítems (Tabla 1).

Tabla 1. Valores del W de Kendall para la validez de contenido de la escala MEID

\begin{tabular}{|c|c|c|c|c|c|}
\hline Dimensión & Ítem & Suficiencia & Coherencia & Relevancia & Claridad \\
\hline \multirow{10}{*}{$\begin{array}{l}\text { 1. Apoyo a la } \\
\text { autonomía }\end{array}$} & 1.1.A & \multirow[t]{10}{*}{$W$ de Kendall $=1 p=.000$} & $W$ de Kendall $=1 p=.000$ & $\mathrm{~W}$ de Kendall $=1 \mathrm{P}=.000$ & W de Kendall $=1 P=.000$ \\
\hline & 1.1.B & & $W$ de Kendall $=1 p=.000$ & $W$ de Kendall $=1 p=.000$ & $W$ de Kendall= $1 p=.000$ \\
\hline & 1.2.A & & $W$ de Kendall $=1 p=.000$ & $W$ de Kendall $=1 p=.000$ & $W$ de Kendall= $1 p=.000$ \\
\hline & 1.1.B & & $W$ de Kendall $=1 p=.000$ & $W$ de Kendall $=1 p=.000$ & $W$ de Kendall $=1 p=.000$ \\
\hline & 1.3.A & & $W$ de Kendall $=1 p=.000$ & W de Kendall $=.57 p=.001$ & W de Kendall $=.76 p=.013$ \\
\hline & 1.3.B & & $W$ de Kendall $=1 p=.000$ & $W$ de Kendall $=.76 p=.000$ & W de Kendall $=.54 p=.001$ \\
\hline & 1.4.A & & $W$ de Kendall $=.76 p=.000$ & W de Kendall $=.54 p=.001$ & W de Kendall $=.44 p=.004$ \\
\hline & 1.4.B & & $W$ de Kendall $=.76 p=.000$ & W de Kendall $=.54 p=.001$ & W de Kendall $=.76 p=.000$ \\
\hline & $1.5 . \mathrm{A}$ & & W de Kendall $=1 p=.000$ & W de Kendall $=.76 p=.000$ & W de Kendall $=.54 p=.001$ \\
\hline & 1.5.B & & $W$ de Kendall $=1 p=.000$ & W de Kendall $=.54 p=.001$ & W de Kendall $=.44 p=.004$ \\
\hline \multirow{10}{*}{$\begin{array}{l}\text { 2. Apoyo a la } \\
\text { estructura } \\
\text { antes de la } \\
\text { tarea }\end{array}$} & 2.1.A & \multirow[t]{10}{*}{ W de Kendall $=.76 p=.000$} & $W$ de Kendall $=1 p=.000$ & $W$ de Kendall $=1 p=.000$ & W de Kendall $=0.76 p=.000$ \\
\hline & 2.1.B & & $W$ de Kendall $=.76 p=.000$ & W de Kendall $=.76 p=.000$ & $W$ de Kendall= $.57 p=.001$ \\
\hline & 2.2.A & & $W$ de Kendall $=1 p=.000$ & W de Kendall $=1 p=.000$ & W de Kendall= $1 p=.000$ \\
\hline & 2.1.B & & $W$ de Kendall $=1 p=.000$ & $W$ de Kendall= $1 p=.000$ & W de Kendall $=.57 p=.001$ \\
\hline & 2.3.A & & $W$ de Kendall $=.76 p=.000$ & W de Kendall $=.57 p=.001$ & W de Kendall $=.76 p=.000$ \\
\hline & $2.3 . \mathrm{B}$ & & W de Kendall $=.57 p=.001$ & W de Kendall $=.44 p=.004$ & W de Kendall $=.76 p=.001$ \\
\hline & $2.4 . \mathrm{A}$ & & $W$ de Kendall $=.76 p=.000$ & W de Kendall $=.54 p=.001$ & W de Kendall $=.76 p=.000$ \\
\hline & $2.4 . B$ & & $W$ de Kendall $=.57 p=.001$ & W de Kendall $=.57 p=.001$ & W de Kendall $=.57 p=.001$ \\
\hline & 2.5.A & & W de Kendall= $1 p=.000$ & $W$ de Kendall $=.76 p=.000$ & W de Kendall $=.38 p=.009$ \\
\hline & $2.5 . B$ & & $W$ de Kendall $=57 p=.001$ & W de Kendall $=.54 p=.001$ & W de Kendall $=.38 p=.009$ \\
\hline \multirow{13}{*}{$\begin{array}{l}3 \text { Apoyo a la } \\
\text { estructura } \\
\text { durante la } \\
\text { tarea }\end{array}$} & 3.1.A & \multirow[t]{13}{*}{ W de Kendall $=.76 p=.000$} & $W$ de Kendall $=1 p=.000$ & W de Kendall $=1 p=.000$ & W de Kendall $=0.76=.000$ \\
\hline & 3.1.B & & $W$ de Kendall $=.76 p=.000$ & W de Kendall $=.76 p=.000$ & W de Kendall $=.54 p=.001$ \\
\hline & 3.1.C & & $W$ de Kendall $=.76 p=.000$ & W de Kendall $=.76 p=.000$ & W de Kendall $=.33 p=.019$ \\
\hline & 3.2.A & & $W$ de Kendall $=1 p=.000$ & $W$ de Kendall $=.54 p=.001$ & W de Kendall $=.54 p=.001$ \\
\hline & 3.2.B & & $W$ de Kendall $=.76 p=.000$ & W de Kendall $=.54 p=.001$ & W de Kendall $=.38 p=.009$ \\
\hline & 3.2.C & & W de Kendall $=.76 p=.000$ & W de Kendall $=.76 p=.000$ & W de Kendall $=.38 p=.009$ \\
\hline & 3.3.A & & $W$ de Kendall $=.57 p=.001$ & W de Kendall $=.57 p=.001$ & W de Kendall $=22 \mathrm{p}=.079$ \\
\hline & 2.3.B & & $W$ de Kendall $=1 p=.000$ & $W$ de Kendall $=.76 p=.000$ & W de Kendall $=.76 p=.000$ \\
\hline & 3.3.C & & W de Kendall $=.57 p=.001$ & W de Kendall $=.57 p=.001$ & W de Kendall $=.44 p=.004$ \\
\hline & 3.4.A & & W de Kendall $=1 p=.000$ & W de Kendall= $1 p=.000$ & W de Kendall= $1 p=.000$ \\
\hline & $3.4 . \mathrm{B}$ & & $W$ de Kendall $=1 p=.000$ & $W$ de Kendall $=1 p=.000$ & W de Kendall $=1 p=.000$ \\
\hline & 3.5.A & & $W$ de Kendall $=1 p=.000$ & W de Kendall $=1 p=.000$ & W de Kendall $=.76 p=.000$ \\
\hline & $3.5 . B$ & & $W$ de Kendall $=.76 p=.000$ & W de Kendall $=.76 p=.000$ & W de Kendall= $1 p=.000$ \\
\hline
\end{tabular}




\section{Escala observacional del estilo motivador en la educación física}

\begin{tabular}{|c|c|c|c|c|c|}
\hline & $3.5 . \mathrm{C}$ & & $W$ de Kendall $=1 p=.000$ & $W$ de Kendall $=.76 p=.000$ & W de Kendall $=.44 p=.004$ \\
\hline & 3.6.A & & $W$ de Kendall $=1 p=.000$ & $W$ de Kendall $=1 p=.000$ & $W$ de Kendall $=1 p=.000$ \\
\hline & $3.6 . \mathrm{B}$ & & $W$ de Kendall $=1 p=.000$ & $W$ de Kendall $=1 p=.000$ & W de Kendall $=.76 p=.000$ \\
\hline & $3.6 . \mathrm{C}$ & & $W$ de Kendall $=1 p=.000$ & $W$ de Kendall $=1 p=.000$ & $W$ de Kendall $=1 p=.000$ \\
\hline & 3.7.A & & $W$ de Kendall $=1 p=.000$ & $W$ de Kendall $=1 p=.000$ & $W$ de Kendall $=1 p=.000$ \\
\hline & 3.7.B & & $W$ de Kendall $=1 p=.000$ & $W$ de Kendall $=.76 p=.000$ & $W$ de Kendall $=.57 p=.001$ \\
\hline & $3.8 . \mathrm{A}$ & & $W$ de Kendall $=1 p=.000$ & $W$ de Kendall $=.76 p=.000$ & $W$ de Kendall $=.76 p=.000$ \\
\hline & $3.8 . \mathrm{B}$ & & $W$ de Kendall $=1 p=.000$ & $W$ de Kendall $=.76 p=.000$ & $W$ de Kendall $=.76 p=.000$ \\
\hline \multirow{19}{*}{$\begin{array}{l}4 \text { Apoyo a la } \\
\text { relación }\end{array}$} & 4.1.A & \multirow[t]{19}{*}{$W$ de Kendall= $.1 p=.000$} & $W$ de Kendall $=1 p=.000$ & $W$ de Kendall $=.54 p=.001$ & $W$ de Kendall $=0.76=.000$ \\
\hline & 4.1.B & & $W$ de Kendall $=1 p=.000$ & $W$ de Kendall $=.57 p=.001$ & $W$ de Kendall $=.57 p=.001$ \\
\hline & 4.1.C & & $W$ de Kendall $=1 p=.000$ & $W$ de Kendall $=.76 p=.000$ & W de Kendall $=.76 p=.000$ \\
\hline & 4.2.A & & $W$ de Kendall $=.76 p=.000$ & $W$ de Kendall $=.76 p=.000$ & $W$ de Kendall $=.54 p=.001$ \\
\hline & 4.2.B & & $W$ de Kendall $=.76 p=.000$ & $W$ de Kendall $=.57 p=.001$ & $W$ de Kendall $=.54 p=.001$ \\
\hline & 4.2.C & & $W$ de Kendall= $1 p=.000$ & W de Kendall $=1 p=.000$ & $W$ de Kendall $=.76 p=.000$ \\
\hline & 4.3.A & & $\mathrm{W}$ de Kendall $=1 \mathrm{p}=.000$ & $W$ de Kendall $=.76 p=.000$ & $W$ de Kendall= $1 p=.000$ \\
\hline & 4.3.B & & $W$ de Kendall $=1 \mathrm{p}=.000$ & $W$ de Kendall $=.76 p=.000$ & $W$ de Kendall $=.76 p=.000$ \\
\hline & 4.3.C & & $W$ de Kendall $=.76 p=.000$ & $W$ de Kendall $=.76 p=.000$ & $W$ de Kendall $=.38 p=.009$ \\
\hline & 4.4.A & & $W$ de Kendall= $1 p=.000$ & W de Kendall $=1 p=.000$ & $W$ de Kendall= $1 p=.000$ \\
\hline & 4.4.B & & $W$ de Kendall $=1 p=.000$ & $W$ de Kendall $=1 P=.000$ & $W$ de Kendall= $.1 p=.000$ \\
\hline & 4.5.A & & $W$ de Kendall $=1 \mathrm{p}=.000$ & $W$ de Kendall $=.76 p=.000$ & $W$ de Kendall $=.76 p=.000$ \\
\hline & 4.5.B & & $W$ de Kendall $=1 \mathrm{p}=.000$ & $W$ de Kendall $=.76 p=.000$ & $W$ de Kendall $=1 p=.000$ \\
\hline & 4.5.C & & $W$ de Kendall= $.57 p=.001$ & $W$ de Kendall $=.54 p=.001$ & $W$ de Kendall $=.17 p=.158$ \\
\hline & 4.6.A & & $W$ de Kendall $=1 p=.000$ & $W$ de Kendall $=.54 p=.001$ & $W$ de Kendall $=1 p=.000$ \\
\hline & $4.6 . B$ & & $W$ de Kendall $=1 p=.000$ & $W$ de Kendall $=.54 p=.001$ & $W$ de Kendall $=1 p=.000$ \\
\hline & 4.7.A & & $W$ de Kendall $=1 p=.000$ & $W$ de Kendall $=.57 p=.001$ & $W$ de Kendall $=1 p=.000$ \\
\hline & 4.7.B & & $W$ de Kendall $=1 p=.000$ & W de Kendall $=.57 p=.001$ & $W$ de Kendall $=1 \mathrm{p}=.000$ \\
\hline & 4.7.C & & $\mathrm{W}$ de Kendall $=.76 p=.000$ & W de Kendall $=.57 p=.001$ & $\mathrm{~W}$ de Kendall $=.57 \mathrm{P}=.001$ \\
\hline
\end{tabular}

Aquellos valores cuya puntuación no superaba los estándares que la literatura sugiere $(<.40)$ (Schmidt, 1997) se sometieron a una nueva fase de validación y se enviaron a los expertos añadiendo ejemplos aclaratorios que facilitaran su comprensión (Tabla 2).
Tras la revisión posterior, se obtuvieron valores para el W de Kendall entre moderados y fuertes (.41-.60) (Schmidt, 1997). Como todos los ítems alcanzaron valores adecuados, no requirió efectuar a una nueva revisión quedando diseñada y validada la escala MEID (Tabla 3)

Tabla 2. Valores del W de Kendall obtenidos en la segunda revisión de la validez de contenido de la escala MEID y ejemplos aclaratorios.

\begin{tabular}{|c|c|c|c|c|}
\hline Dimensión & Ítems & Coherencia & Relevancia & Claridad \\
\hline \multirow{4}{*}{$\begin{array}{l}\text { 1.Apoyo } \\
\text { a la } \\
\text { Autonomía }\end{array}$} & $\begin{array}{l}\text { 1.4.A. Ofrece posibilidades de experimentación (individualiza la } \\
\text { enseñanza) }\end{array}$ & $\begin{array}{l}\text { W de Kendall }=.76 \\
p=.000\end{array}$ & $\begin{array}{l}\text { W de Kendall }=.54 \\
p=.001\end{array}$ & $\begin{array}{l}\text { W de Kendall }=54 \\
p=.001\end{array}$ \\
\hline & \multicolumn{4}{|c|}{$\begin{array}{l}\text { Dando soporte de autonomía: Por medio del descubrimiento propone variantes para reforzar o ampliar según sus posibilidades. Tras } \\
\text { un saque de mano baja que no pasa la red, les pregunta “¿Dónde pensáis que falláis? ¿Cómo podríamos solucionarlo? ¿Qué podríamos } \\
\text { hacer para mejorar? (refuerzo y afianzamiento)”. Mientras que en otro grupo que logra el objetivo (pasar la red) les propone “¿podríais } \\
\text { sacar de otra manera? ¿Sabríais dirigir el saque con una intencionalidad concreta? (ampliación). }\end{array}$} \\
\hline & $\begin{array}{l}\text { 1.5.B. No cede la responsabilidad. (El docente impone las } \\
\text { tareas de forma que genera presión emocional e inhibe al } \\
\text { estudiante) }\end{array}$ & $\begin{array}{l}\text { W de Kendall }=1 \\
p=.000\end{array}$ & $\begin{array}{l}\text { W de Kendall }=.54 \\
p=.001\end{array}$ & $\begin{array}{l}\text { W de Kendall }=.57 \\
p=.001\end{array}$ \\
\hline & \multicolumn{4}{|c|}{$\begin{array}{l}\text { En forma controladora: "Nadie empieza hasta que yo prepare el material y mientras tanto, todo el mundo en su sitio sin moverse"; } \\
\text { "fijaros cómo hago los ejercicios que luego van a examen y el que no los haga como yo no aprobará". }\end{array}$} \\
\hline Dimensión & Ítems & Coherencia & Relevancia & Claridad \\
\hline \multirow{2}{*}{$\begin{array}{l}\text { 2. Apoyo } \\
\text { a la } \\
\text { estructura } \\
\text { antes de la } \\
\text { tarea }\end{array}$} & $\begin{array}{l}\text { 2.5.A. Ofrece pautas y orientaciones para regular el progreso } \\
\text { personal y da a conocer previamente los criterios de mejora (El } \\
\text { docente deja claro qué deben hacer para mejorar y } \\
\text { proporcionar indicaciones para lograrlo }\end{array}$ & $\begin{array}{l}\text { W de Kendall = } 1 \\
p=.000\end{array}$ & $\begin{array}{l}\text { W de Kendall }=.76 \\
p=.000\end{array}$ & $\begin{array}{l}\text { W de Kendall }=.54 \\
p=.001\end{array}$ \\
\hline & \multicolumn{4}{|c|}{$\begin{array}{l}\text { Dando soporte de autonomía: Explica cómo autoevaluar su propio progreso. "Aquí tenéis las fichas para trabajar las capacidades físicas. } \\
\text { Cada una contiene unos indicadores con unos niveles asociados. Esto os permitirá saber en qué nivel estáis actualmente y cómo poder }\end{array}$} \\
\hline
\end{tabular}




\section{Barrachina-Peris et al.}

\begin{tabular}{|c|c|c|c|c|}
\hline & \multicolumn{4}{|c|}{$\begin{array}{l}\text { mejorar. Si utilizáis el registro personal, podréis ir comparando cómo vais en cada clase y reflexionar sobre el porqué de cada } \\
\text { realización". }\end{array}$} \\
\hline & $\begin{array}{l}\text { 2.5.B. No ofrece pautas ni orientaciones a modo de indicador } \\
\text { de mejora. }\end{array}$ & $\begin{array}{l}\text { W de Kendall }=.57 \\
p=.001\end{array}$ & $\begin{array}{l}\text { W de Kendall }=.54 \\
p=.001\end{array}$ & $\begin{array}{l}\text { W de Kendall }=.54 \\
p=.001\end{array}$ \\
\hline & $\begin{array}{l}\text { En forma controladora: "Hay que lograr } 20 \text { repeticiones para a } \\
\text { que la semana que viene es la prueba práctica". }\end{array}$ & robar, dispondréis de & n intento por person & a. Aprovechad el tiempo \\
\hline Dimensión & Ítems & Coherencia & Relevancia & Claridad \\
\hline \multirow{16}{*}{$\begin{array}{l}\text { 3. Apoyo } \\
\text { a la } \\
\text { estructura } \\
\text { durante la } \\
\text { tarea }\end{array}$} & $\begin{array}{l}\text { 3.1.C. Describe la situación sin aludir a los participantes sin } \\
\text { promover el progreso ni emplear un lenguaje controlador. }\end{array}$ & $\begin{array}{l}\text { W de Kendall }=.76 \\
p=.000\end{array}$ & $\begin{array}{l}\text { W de Kendall }=.76 \\
p=.000\end{array}$ & $\begin{array}{l}\text { W de Kendall }=.54 \\
p=.001\end{array}$ \\
\hline & \multicolumn{4}{|l|}{ En forma neutra: "Veo que estáis practicando por parejas el bloqueo" } \\
\hline & $\begin{array}{l}\text { 3.2.B Utiliza los modelos a través de estudiantes y emplea un } \\
\text { lenguaje controlador (emplea un tono que evidencia } \\
\text { menosprecio). }\end{array}$ & $\begin{array}{l}\text { W de Kendall }=.76 \\
p=.000\end{array}$ & $\begin{array}{l}\text { W de Kendall }=.54 \\
p=.001\end{array}$ & $\begin{array}{l}\text { W de Kendall }=.57 \\
p=.001\end{array}$ \\
\hline & \multicolumn{4}{|c|}{$\begin{array}{l}\text { En forma controladora: Destaca los errores producidos en la ejecución y utiliza un lenguaje que menosprecia/minimiza los puntos } \\
\text { fuertes "¿Eso es un pase? ¿Es así como he explicado que se hacían los pases? Pon las manos estiradas que parece que tengas miedo a } \\
\text { que te muerdan ¿quieres estirar las manos para cortar el pase? iQue no te va a morder!”. }\end{array}$} \\
\hline & $\begin{array}{l}\text { 3.2.C. Utiliza modelos sin aportar información, ni positiva ni } \\
\text { negativa y sin emplear un lenguaje controlador }\end{array}$ & $\begin{array}{l}\text { W de Kendall }=.76 \\
p=.000\end{array}$ & $\begin{array}{l}\text { W de Kendall }=.76 \\
p=.000\end{array}$ & $\begin{array}{l}\text { W de Kendall }=.54 \\
p=.001\end{array}$ \\
\hline & \multicolumn{4}{|c|}{ En forma neutra: Propone la situación de 2×2, pide ejecutarla y afirma "aquí tenéis un ejemplo". } \\
\hline & $\begin{array}{l}\text { 3.3.A Cuando es necesario comparte con los estudiantes las } \\
\text { demostraciones }\end{array}$ & $\begin{array}{l}\text { W de Kendall }=.57 \\
p=.001\end{array}$ & $\begin{array}{l}\text { W de Kendall }=.57 \\
p=.001\end{array}$ & $\begin{array}{l}\text { W de Kendall }=.54 \\
p=.001\end{array}$ \\
\hline & \multicolumn{4}{|c|}{$\begin{array}{l}\text { Dando soporte de autonomía: En una situación de deporte de lucha, comparte la demostración con un estudiante dándole las } \\
\text { indicaciones para que tome decisiones más acertadas. "Yo me tumbaré y tú me coges por la cintura y me bloqueas la cadera, ¿¿Mi } \\
\text { ejemplo te sirve para ver cómo hay que hacerlo?, ¿Lo pruebas ahora con tu compañero a ver qué tal? }\end{array}$} \\
\hline & $\begin{array}{l}\text { 3.3.B. No participa en las demostraciones. Las dirige usando un } \\
\text { lenguaje controlador. }\end{array}$ & $\begin{array}{l}\text { W de Kendall }=1 \\
p=.000\end{array}$ & $\begin{array}{l}\text { W de Kendall }=.76 \\
p=.000\end{array}$ & $\begin{array}{l}\text { W de Kendall }=.76 \\
p=.000\end{array}$ \\
\hline & \multicolumn{4}{|c|}{$\begin{array}{l}\text { En forma controladora: "Quieres cogerlo por la cintura y presionarlo con tu cuerpo para que no se levante, iqué no es un abrazo!, } \\
\text { ¿no tienes más fuerza?". }\end{array}$} \\
\hline & $\begin{array}{l}\text { 3.3.C. No participa en las demostraciones, e interviene sin } \\
\text { informar al respecto. }\end{array}$ & $\begin{array}{l}\text { W de Kendall }=.57 \\
p=.001\end{array}$ & $\begin{array}{l}\text { W de Kendall }=.57 \\
p=.001\end{array}$ & $\begin{array}{l}\text { W de Kendall }=.54 \\
p=.001\end{array}$ \\
\hline & \multicolumn{4}{|l|}{ En forma neutra: ¿Cuántos intentos lleváis? } \\
\hline & $\begin{array}{l}\text { 3.6.C. Ofrece feed-backs neutros y ambiguos que no aportan } \\
\text { nada sobre el desarrollo de la acción. }\end{array}$ & $\begin{array}{l}\text { W de Kendall }=1 \\
p=.000\end{array}$ & $\begin{array}{l}\text { W de Kendall }=.76 \\
p=.000\end{array}$ & $\begin{array}{l}\text { W de Kendall }=.54 \\
p=.001\end{array}$ \\
\hline & \multicolumn{4}{|c|}{$\begin{array}{l}\text { En forma neutra: Se dirige a los estudiantes sin reforzar ni positiva ni negativamente. "Veo que estáis en fila esperando vuestro turno, } \\
\text { os queda tiempo para seguir practicando". }\end{array}$} \\
\hline & 3.8.B. No fomenta agrupaciones flexibles. & $\begin{array}{l}\text { W de Kendall }=1 \\
p=.000\end{array}$ & $\begin{array}{l}\text { W de Kendall }=.76 \\
p=.000\end{array}$ & $\begin{array}{l}\text { W de Kendall }=.76 \\
p=.000\end{array}$ \\
\hline & \multicolumn{4}{|c|}{$\begin{array}{l}\text { En forma controladora: Impone las agrupaciones, formando los equipos con su criterio personal o al azar pero siempre dirigido por el } \\
\text { docente. "Los números pares a mi derecha y los impares a mi izquierda. Los cinco primeros de la lista aquí y van a jugar contra los cinco } \\
\text { siguientes". }\end{array}$} \\
\hline Dimensión & Ítems & Coherencia & Relevancia & Claridad \\
\hline \multirow{4}{*}{$\begin{array}{l}\text { 4. Apoyo a } \\
\text { la } \\
\text { relación }\end{array}$} & 4.3.C. Les escucha pero no atiende a las dudas o las pospone. & $\begin{array}{l}\text { W de Kendall }=.76 \\
p=.000\end{array}$ & $\begin{array}{l}\text { W de Kendall }=.76 \\
p=.001\end{array}$ & $\begin{array}{l}\text { W de Kendall }=.57 \\
p=.001\end{array}$ \\
\hline & \multicolumn{4}{|c|}{$\begin{array}{l}\text { En forma neutra Los estudiantes se dirigen al docente y le dicen que no saben saltar, el docente les escucha, pero cambia de tarea. "No } \\
\text { sabemos saltar a la comba", a lo que responde, "cambio, nos colocamos en la siguiente estación" o "luego lo vemos" }\end{array}$} \\
\hline & 4.5.C. No muestra entusiasmo ni indiferencia & $\begin{array}{l}\text { W de Kendall }=.57 \\
p=.001\end{array}$ & $\begin{array}{l}\text { W de Kendall }=.54 \\
p=.001\end{array}$ & $\begin{array}{l}\text { W de Kendall }=54 \\
p=.001\end{array}$ \\
\hline & \multicolumn{4}{|l|}{ En forma neutra: En este grupo falta uno, ¿alguien se pone? } \\
\hline
\end{tabular}




\section{Escala observacional del estilo motivador en la educación física}

Tabla 3. Medida del Estilo Interpersonal Docente (MEID)

\begin{tabular}{|c|c|}
\hline Categoría & Subcategoría \\
\hline \multirow[t]{5}{*}{$\begin{array}{l}\text { 1. Apoyo a la } \\
\text { autonomía }\end{array}$} & $\begin{array}{l}\text { Contexto: En el transcurso de la clase cuando el docente propone una situación de enseñanza-aprendizaje... } \\
\text { 1.1.A. Dando soporte de autonomía: Pregunta al estudiante sobre sus preferencias en relación a una tarea. } \\
\text { 1.1.B. En forma controladora: No pregunta ni tiene en cuenta las preferencias del estudiante. }\end{array}$ \\
\hline & $\begin{array}{l}\text { Contexto: En el transcurso de la clase, el docente cuando plantea las tareas... } \\
\text { 1.2.A. Dando soporte autonomía: Ofrece posibilidad de elección al estudiante (agrupaciones, materiales y espacios). } \\
\text { 1.2.B. En forma controladora: No ofrece elección al estudiante. Todo está definido previamente por el criterio del } \\
\text { docente. }\end{array}$ \\
\hline & $\begin{array}{l}\text { Contexto: El docente plantea las tareas de forma que los estudiantes puedan tomar decisiones acerca de su } \\
\text { intervención... } \\
\text { 1.3.A. Dando soporte de autonomía: Deja que el estudiante tome la iniciativa (cede la iniciativa). } \\
\text { 1.3.B. En forma controladora: No cede la iniciativa. Les informa sobre cuándo ejecutar las tareas sin dejar opción. }\end{array}$ \\
\hline & $\begin{array}{l}\text { Contexto: El docente, cuando plantea las tareas, da opciones para afianzar, ampliar o reforzar los objetivos } \\
\text { perseguidos. } \\
\text { 1.4.A. Dando soporte de autonomía: Ofrece posibilidades de experimentación (individualiza la enseñanza). } \\
\text { 1.4.B. En forma controladora: Propone tareas cerradas y anticipa las respuestas. }\end{array}$ \\
\hline & $\begin{array}{l}\text { Contexto: El docente manifiesta expectativas positivas hacia el grupo y organiza las tareas de forma que... } \\
\text { 1.5.A. Dando soporte de autonomía: Cede responsabilidad. } \\
\text { 1.5.B. En forma controladora: No cede responsabilidad, genera presión emocional e inhibe al estudiante. }\end{array}$ \\
\hline \multirow[t]{5}{*}{$\begin{array}{l}\text { 2. Apoyo a la } \\
\text { estructura antes de la } \\
\text { tarea }\end{array}$} & $\begin{array}{l}\text { Contexto: El docente habitualmente informa a los estudiantes... } \\
\text { 2.1.A. Dando soporte de autonomía: Al iniciar la clase explica y razona los objetivos. } \\
\text { 2.1.B. En forma controladora: No explica los objetivos y se centra en el contenido. }\end{array}$ \\
\hline & $\begin{array}{l}\text { Contexto: Antes de comenzar la práctica el docente... } \\
\text { 2.2.A. Dando soporte de autonomía: Explica la estructura global de la clase (la organización y la finalidad). } \\
\text { 2.2.B. En forma controladora: No explica la estructura previamente. Llegado el momento, expone las tareas y asigna a } \\
\text { los estudiantes. }\end{array}$ \\
\hline & $\begin{array}{l}\text { Contexto: El docente ofrece argumentos sobre la transferencia social que tiene la realización de una actividad... } \\
\text { 2.3.A. Dando soporte de autonomía: Explica la utilidad de las tareas. } \\
\text { 2.3.B. En forma controladora: No explica la utilidad de las tareas y se centra en la ejecución. }\end{array}$ \\
\hline & $\begin{array}{l}\text { Contexto: El docente, cuando necesita ilustrar antes de empezar la clase... } \\
\text { 2.4.A. Dando soporte de autonomía: Se apoya en los estudiantes como modelos positios para realizar demostraciones. } \\
\text { 2.4.B. En forma controladora: No se apoya en los estudiantes, el docente es el modelo a imitar. }\end{array}$ \\
\hline & $\begin{array}{l}\text { Contexto: En el transcurso de las clases, el docente recuerda para qué se plantean las tareas... } \\
\text { 2.5.A. Dando soporte de autonomía: Ofrece pautas y orientaciones para regular el progreso personal y da a conocer } \\
\text { previamente los criterios de mejora. } \\
\text { 2.5.B. En forma controladora: No ofrece pautas ni orientaciones a modo de indicador de mejora. }\end{array}$ \\
\hline \multirow[t]{6}{*}{$\begin{array}{l}\text { 3. Apoyo a la } \\
\text { estructura durante la } \\
\text { tarea }\end{array}$} & $\begin{array}{l}\text { Contexto: El docente durante la ejecución de las actividades... } \\
\text { 3.1.A. Dando soporte de autonomía: Adapta las instrucciones según el progreso de los estudiantes. } \\
\text { 3.1.B. En forma controladora: Mantiene constantes las informaciones, con independencia del progreso (empleando un } \\
\text { lenguaje controlador). } \\
\text { C. En forma neutra: El docente únicamente describe la situación sin aludir a los participantes sin promover el progreso } \\
\text { ni emplear un lenguaje controlador. }\end{array}$ \\
\hline & $\begin{array}{l}\text { Contexto: El docente cuando necesita ilustrar las tareas una vez iniciadas... } \\
\text { 3.2.A. Dando soporte de autonomía: Utiliza a modelos a través de estudiantes. } \\
\text { 3.2.B. En forma controladora: Utiliza los modelos a través de estudiantes y emplea un lenguaje controlador (emplea un } \\
\text { tono que evidencia menosprecio). } \\
\text { C. En forma neutra: Utiliza modelos sin aportar información, ni positiva ni negativa. }\end{array}$ \\
\hline & $\begin{array}{l}\text { Contexto: El docente participa en las explicaciones de las tareas... } \\
\text { 3.3.A. Dando soporte de autonomía: Cuando es necesario comparte con los estudiantes las demostraciones. } \\
\text { 3.3.B. En forma controladora: No participa en las demostraciones. Las dirige usando lenguaje controlador. } \\
\text { C. En forma neutra: No participa en las demostraciones e interviene sin informar al respecto. }\end{array}$ \\
\hline & $\begin{array}{l}\text { Contexto: El docente cuando propone una actividad... } \\
\text { 3.4.A. Dando soporte de autonomía: Propone diferentes variantes para una misma tarea. } \\
\text { 3.4.B. En forma controladora: No ofrece variantes para una misma tarea, sino que impone su visión como única opción. }\end{array}$ \\
\hline & $\begin{array}{l}\text { Contexto: Durante la realización de las tareas, el docente... } \\
\text { 3.5.A. Dando soporte de autonomía: Ofrece refuerzos positivos tanto verbales como no verbales. Anima a los } \\
\text { estudiantes a que perseveren. } \\
\text { 3.5.B. En forma controladora: Destaca y penaliza las ejecuciones incorrectas } \\
\text { C. En forma neutra: Se limita a describir una situación sin emplear un lenguaje controlador. Las interacciones que } \\
\text { proporciona no son positivas ni negativas. }\end{array}$ \\
\hline & $\begin{array}{l}\text { Contexto: El docente, durante el transcurso de las actividades... } \\
\text { 3.6.A. Dando soporte de autonomía: Ofrece feed-backs informativos y/o positivos durante la ejecución de las tareas } \\
\text { 3.6.B. En forma controladora: No ofrece feed-backs informativos ni positivos, únicamente negativos. }\end{array}$ \\
\hline
\end{tabular}




\section{Barrachina-Peris et al.}

\begin{tabular}{|c|c|}
\hline & 3.6.C. En forma neutra: Ofrece feed-backs neutros y ambiguos que no aportan nada sobre el desarrollo de la acción. \\
\hline & $\begin{array}{l}\text { Contexto: El docente plantea las actividades habitualmente de forma que... } \\
\text { 3.7.A. Dando soporte de autonomía: Ofrece una graduación de la dificultad de las tareas según el nivel de los } \\
\text { estudiantes } \\
\text { 3.7.B. En forma controladora: Gradúa la dificultad de las tareas según a la norma y no al nivel del estudiante. }\end{array}$ \\
\hline & $\begin{array}{l}\text { Contexto: En el transcurso de las actividades, el docente... } \\
\text { 3.8.A. Dando soporte de autonomía: Propone agrupaciones flexibles según el desarrollo de las tareas. } \\
\text { 3.8.B. En forma controladora: No fomenta agrupaciones flexibles. }\end{array}$ \\
\hline \multirow[t]{7}{*}{ 4. Apoyo a la relación } & $\begin{array}{l}\text { Contexto: Durante en el transcurso de la sesión, el docente... } \\
\text { 4.1.A. Dando soporte de autonomía: Se dirige a los estudiantes con educación y de manera individualizada. } \\
\text { 4.1.B. En forma controladora: Se dirige a los estudiantes individualmente o en gran grupo de forma imperativa. } \\
\text { 4.1.C. En forma neutra: Se dirige a los estudiantes en gran grupo empleando un lenguaje impersonal, inespecífico. }\end{array}$ \\
\hline & $\begin{array}{l}\text { Contexto: El docente en clase cuando se dirige a los estudiantes... } \\
\text { 4.2.A. Dando soporte de autonomía: Emplea un lenguaje empático } \\
\text { 4.2.B. En forma controladora: No emplea un lenguaje empático y no se adapta al estudiante. } \\
\text { 4.2.C. En forma neutra: Utiliza un lenguaje apático cuando se dirige a los estudiantes (sin emoción, no aporta nada ni } \\
\text { positivo ni negativo). }\end{array}$ \\
\hline & $\begin{array}{l}\text { Contexto: Cuando los estudiantes se dirigen al docente, éste... } \\
\text { 4.3.A. Dando soporte de autonomía: Escucha a los estudiantes con actitud activa y positiva. } \\
\text { 4.3.B. En forma controladora: No tiene una actitud de escucha activa. } \\
\text { 4.3.C. En forma neutra: Les escucha, pero no atiende a sus dudas o las pospone. }\end{array}$ \\
\hline & $\begin{array}{l}\text { Contexto: El docente durante en el transcurso de la clase... } \\
\text { 4.4.A. Dando soporte de autonomía: Se aproxima al estudiante para atenderle. } \\
\text { 4.4.B. En forma controladora: No se aproxima para atender a los estudiantes. }\end{array}$ \\
\hline & $\begin{array}{l}\text { Contexto El docente cuando interactúa en clase... } \\
\text { 4.5.A. Dando soporte de autonomía: Es entusiasta. } \\
\text { 4.5.B. En forma controladora: No muestra entusiasmo. } \\
\text { 4.5.C. En forma neutra: Ni muestra entusiasmo ni indiferencia. }\end{array}$ \\
\hline & $\begin{array}{l}\text { Contexto El docente durante en el transcurso de la clase... } \\
\text { 4.6.A. Dando soporte de autonomía: Da confianza a los estudiantes. } \\
\text { 4.6.B. En forma controladora: No da confianza y genera inseguridad en clase. }\end{array}$ \\
\hline & $\begin{array}{l}\text { Contexto El docente durante en el transcurso de la clase... } \\
\text { 4.7.A. Dando soporte de autonomía: Se comporta como un modelo positivo para los estudiantes. } \\
\text { 4.7.B. En forma controladora: No se comporta como un modelo positivo para los estudiantes. } \\
\text { 4.7.C. En forma neutra: No se comporta ni positiva ni negativamente, guarda silencio mientras los participantes } \\
\text { ejecutan la tarea y si interviene lo hace para describir algo que está fuera de la ejecución, como las repeticiones o el } \\
\text { tiempo que llevan o falta para terminar. }\end{array}$ \\
\hline
\end{tabular}

\section{Etapa final}

Tercera fase. Validez de comprensión del instrumento de observación. La validez de comprensión se llevó a cabo con una muestra independiente compuesta por 10 docentes universitarios. Se registraron las preguntas, dudas y sugerencias que realizaron y se analizaron con la técnica Delphi. Los ítems no sufrieron modificación alguna ya que alcanzaron valores próximos a 1 , siendo ésta la versión final (Tabla 3). Los índices de fiabilidad inter observadores (grupo de expertos/ grupo coordinador) se obtuvieron al alcanzar un consenso total y la fiabilidad intra observadores (tanto en grupo de expertos como en el grupo coordinador) se logró al obtener valores adecuados en el coeficiente de correlación intraclase (Portney y Walkings, 2009).

La validez del instrumento se comprobó a través de un primer estudio piloto. Durante 8 clases, dos grupos implementaron los mismos contenidos. El grupo control ( $n$ $=28 ; M=13.4$ años; $D T=1.1$ ) siguió una metodología de corte controlador, mientras que el grupo experimental $(n=$ 26; $M=13.5$ años; $D T=0.8$ ) desarrolló la instrucción a través del apoyo a la autonomía. En ambos grupos se empleó la escala para medir el estilo interpersonal motivador que el docente utilizaba durante la instrucción, arrojando datos válidos. La escala demostró ser válida en los dos escenarios de enseñanza-aprendizaje, puesto que midió correctamente los diferentes estilos y ser fiable, ya que se filmaron en vídeo las clases y se analizaron por varios observadores obteniéndose valores consistentes (Tabla 4).

Tabla 4. Evolución de los porcentajes del estilo interpersonal docente por grupo.

\begin{tabular}{ccccccc}
\hline & \multicolumn{3}{c}{$\begin{array}{c}\text { Grupo apoyo a la } \\
\text { autonomía }\end{array}$} & \multicolumn{3}{c}{$\begin{array}{c}\text { Grupo estilo } \\
\text { controlador }\end{array}$} \\
\hline & AA & EC & EN & AA & EC & EN \\
& $(\%)$ & $(\%)$ & $(\%)$ & $(\%)$ & $(\%)$ & $(\%)$ \\
\hline Clase 1 & 85 & 0 & 15 & 0 & 90 & 10 \\
Clase 2 & 87 & 0 & 13 & 0 & 91 & 9 \\
Clase 3 & 95 & 0 & 5 & 0 & 92 & 8 \\
Clase 4 & 95 & 0 & 5 & 0 & 85 & 15 \\
Clase 5 & 93 & 0 & 7 & 0 & 94 & 6 \\
Clase 6 & 98 & 0 & 2 & 0 & 88 & 12 \\
Clase 7 & 97 & 0 & 3 & 0 & 92 & 8 \\
Clase 8 & 96 & 0 & 4 & 0 & 89 & 11 \\
\hline
\end{tabular}




\section{Escala observacional del estilo motivador en la educación física}

\section{DISCUSIÓN}

El objetivo del estudio fue el de desarrollar una escala de estimación para el desarrollo del estilo motivacional docente, y aportar evidencias de su fiabilidad y validez. Se revisaron las escalas encontradas en la literatura y se analizaron sus fortalezas y limitaciones. Los instrumentos existentes han permitido medir el estilo interpersonal motivador que el docente despliega en la instrucción desde una perspectiva ecológica. Sin embargo, el procedimiento para medir y registrar los comportamientos, la interpretación, categorización de éstos y la confrontación entre estilos, siguen siendo sus principales limitaciones (Jiang et al., 2019). Considerando las limitaciones de los instrumentos revisados, se diseñó un instrumento que midiera el estilo interpersonal motivador docente como dos factores excluyentes. A partir de los escenarios propuestos por Reeve (2009), Reeve et al. (2014) y Sarrazin, Tessier, Pelletier, Trouilloud, y Chanal (2006) y apoyado en el instrumento de Haerens et al. (2013), se diseñó una escala para medir las interacciones durante el planteamiento y desarrollo de las tareas. Se adoptó la tarea como unidad de análisis, atendiendo a criterios instructivos. La tarea representa la unidad significante mínima de la instrucción y del clima desplegado por el docente en clase, convirtiéndose en un indicador válido para determinar la calidad de la enseñanza o calidad docente (León et al., 2018) al obtenerse una matriz de análisis que corresponde a cada dimensión de la escala de estimación.

El instrumento fue diseñado empleando la técnica Delphi y fue sometido a un proceso de validación a través de la técnica de la validez de contenido. Diversos expertos fueron solicitados para analizar el grado de suficiencia, coherencia, relevancia y claridad. Tras varias revisiones, se obtuvieron índices adecuados en el W Kendall, los cuales indican que la escala es válida y fiable para medir el apoyo a la autonomía como un factor independiente del estilo controlador o el estilo neutro.

Los resultados de este estudio revelaron que la estructura de la escala de medida del estilo interpersonal motivador docente apoya el modelo de cuatro factores. El constructo diseñado quedó compuesto por 60 reactivos o unidades de observación estructurados en cuatro dimensiones o categorías exhaustivas y mutuamente excluyentes: apoyo a la autonomía (interacciones docentes encaminadas a que el estudiante movilice sus recursos internos), apoyo a la estructura antes de la tarea (clarificación de los objetivos y la organización de la clase en base a éstos), apoyo a la estructura durante la tarea (feedback informativo proporcionado por el docente durante la instrucción) y apoyo a la relación (interacción afectuosa y de respeto mutuo con los estudiantes y entusiasmo mostrado por el docente durante la instrucción). Este resultado está en consonancia con estudios anteriores en contextos educativos (Haerens et al., 2013; Krijgsman et al., 2019) y sugiere que está podría ser la línea a seguir para analizar el estilo motivador docente de apoyo a la autonomía durante la instrucción.

\section{CONCLUSIONES}

Estos resultados proporcionan evidencia de la solidez de la estructura de esta medida y revelan que esta versión consigue replicar fielmente la estructura teórica subyacente. Así pues, en lo sucesivo, la escala de medida del estilo interpersonal motivador docente puede servir para medir el estilo interpersonal motivador. No obstante, la escala de medida del estilo interpersonal motivador docente presenta limitaciones a nivel cuantitativo y cualitativo. La cantidad de conductas analizadas pueden dificultar una medida ágil, sobre todo en las primeras fases. Además, la utilización de la frecuencia como parámetro primario de registro puede presentar algunas limitaciones dentro de la metodología observacional (Anguera, 1990). Por otro lado, los resultados de la validez de contenido, revelaron que algunos ítems presentaban poca claridad, generando confusión para interpretarlos. En este sentido, se sugieren dos estrategias. Por un lado, revisar algunos ítems planteados para minimizar la ambigüedad que puedan dar lugar y por otro, diseñar una versión corta de la escala y validarla, que permita una codificación más ágil.

\section{APLICACIONES PRÁCTICAS}

La escala MEID, debido a sus índices adecuados de fiabilidad y validez, además de permitir la observación del estilo motivador docente en las situaciones de instrucción, podría ser empleada para la formación de docentes en el estilo interpersonal motivador de apoyo a la autonomía, puesto que representa un sistema de categorías exhaustivo y mutuamente excluyente que hace posible establecer un perfil motivador específico, a partir del cual se pueden establecer criterios para formar al docente en un estilo motivador de apoyo a la autonomía. A su vez, la propia naturaleza del estilo motivador que promueve la escala (ceder responsabilidades y promover las iniciativas de los estudiantes, entre otras) podría ser útil para diseñar ambientes de aprendizaje creativos/novedosos, que fomentaran una mayor implicación y motivación intrínseca de los estudiantes, ya que las interacciones de la escala contemplan la tolerancia al error como una estrategia clave para minimizar el control indirecto que determinadas conductas docentes ejercen sobre el aprendizaje y bienestar de los estudiantes (Jiang et al., 2019). La estructura que adopta la escala puede ayudar a un análisis de la interacción docente más cercano a la realidad. En definitiva, la escala es un instrumento que puede servir para mejorar la calidad docente porque pretende comprender el estilo comportamental durante la interacción del docente en la 


\section{Barrachina-Peris et al.}

instrucción (apoyo a la autonomía, controlador y neutro), entendido como un factor mediador de primer orden para satisfacer o atender a de las necesidades básicas de los estudiantes y para desencadenar una implicación en las tareas de forma autodeterminada.

\section{AGRADECIMIENTOS}

Este trabajo ha sido desarrollado en el marco de los proyectos de investigación $\mathrm{I}+\mathrm{D}+\mathrm{i}$ correspondiente al programa estatal de investigación, desarrollo e innovación orientada a los Retos de la Sociedad DEP2017-83441-R subvencionado por el Ministerio de Economía, Industria y Competitividad.

\section{REFERENCIAS}

1. Abós, A., Sevil, J., Martín-Albo, L., Julián, J.A., y García-González, L. (2018). An integrative framework to validate the need-supportive teaching style scale (NSTSS) in secondary teachers through exploratory structural equation modeling. Contemporay Educational Psychology, 52, 48-60. http://doi.org/10.1016/j.cedpsych.2018.01.001

2. Aelterman, N., Vansteenkiste, M., Haerens, L., Soenens, B., Fontaine, J.R.J., y Reeve, J. (2018). Toward an integrative and fine-grained insight in motivating and demotivating teachin styles: the merits of a circumplex approach. Journal of Educational Psychology, 111(3), 497-521. http://dx.doi.org/10.1037/edu0000293

3. Aguado-Gómez, R., Díaz-Cueto, M., HernándezÁlvarez, J.L., y López-Rodríguez, A. (2016). Apoyo a la autonomía en las clases de educación física: percepción versus realidad. Ciencias de la Actividad Física y el Deporte, 16(62), 183-202. http://dx.doi.org/10.15366/rimcafd2016.62.001

4. Anguera, M. T. (1990). Metodología observacional. En J. Arnau, M. T. Anguera y J. Gómez. Metodología de la investigación en Ciencias del Comportamiento (pp. 125-236). Murcia: Secretariado de Publicaciones de la Universidad de Murcia.

5. Anguera, M.T., Blanco-Villaseñor, A., HernándezMendo, A. y Losada, J.L. (2011). Diseños Observacionales: ajuste y aplicación en psicología del deporte. Cuadernos de Psicología del Deporte, 11(2) 63-76. 84232015000100002

6. Anguera, M.T., Camerino, O., Castañer, M., y Sánchez-Algarra (2014): Mixed Methods en la investigación de la actividad física y el deporte. Revista de Psicología del Deporte, 23(1), 123-130. https://doi.org/10.4321/s1578-84232015000100008

7. Anguera, M.T., y Hernández-Mendo, A. (2013). La metodología observacional en el ámbito del deporte. $E$ balonmano.com: Revista de Ciencias del Deporte 9(3), 135-160. 84232015000100002

https://doi.org/10.4321/s1578-

8. Anguera, M.T., y Hernández-Mendo, A. (2014): Técnicas de análisis en estudios observacionales en ciencias del deporte. Cuadernos de Psicología del Deporte, 15(1) 13-30. https://doi.org/10.4321/s157884232015000100002

9. Anguera, M.T., Blanco-Villaseñor, A., Losada, J.L., y Hernández-Mendo, A. (2000). Metodología observacional en psicología en el deporte: conceptos básicos. Lecturas: EF y Deportes. Revista Digital, 24, agosto 2000. http://www.efdeportes.com/efd24b/obs.htm [Consulta: 10 de octubre de 2000].

10. Anguera. M.T., Blanco-Villaseñor, A., Losada, J.L., y Portell, M (2018). Pautas para elaborar trabajos que utilizan la metodología observacional. Anuario de psicología, 14(1), 9-17. https://doi.org/10.1016/j.anpsic.2018.02.001

11. Assor, A., Kaplan, H., y Roth, G. (2002). Choice is good, but relevance is excellent: Autonomy-enhancing and suppressing teaching behaviors predicting students' engagement in schoolwork. British Journal of Educational Psychology, 27, 261-278. https://doi.org/10.1348/000709902158883

12. Cheon, S. H., y Reeve, J. (2013). Do the benfits from autonomy-supportive $\mathrm{PE}$ teacher training programs endure? A one-year follow-up investigation. Psychology of Sport and Exercise, 14, 508-518. http://dx.doi.org/10.1016/j.psychsport.2013.02.002

13. Cheon, S. H., y Reeve, J. (2015). A classroom-based intervention to help teachers to decrease students' amotivation. Contemporanean Educational Psychology, 40, 99-111. http://dx.doi.org/10.1016/j.cedpsych.2014.06.004

14. Cheon, S. H., Reeve, J., y Moon, I. S. (2012). Experimentally based, longitudinally designed, teacher-focused intervention to help physical education teachers be more autonomy supportive toward their studens. Journal of Sport and Exercise Psychology, 34, 365-396. https://doi.org/10.1123/jsep.34.3.365

15. Cheon, S. H., Reeve, J., y Ntoumanis, N. (2018). A needs-supportive intervention to help $\mathrm{PE}$ teachers enhance students' prosocial behavior and diminish antisocial behavior. Psychology of Sport and Exercise, 35, 74-88. http://dx.doi.org/10.1016/j.psychsport.2017.11.010

16. Cheon, S. H., Reeve, J., y Song (2016). A teacher focused intervention to decrease PE students' amotivation by increasing need satisfaction and 


\section{Escala observacional del estilo motivador en la educación física}

decreasing need fustration. Journal of Sport and Exercise, 38(3), 217-235. http://dx.doi.org/10.1123/jsep.2015-0236

17. Cheon, S. H., Reeve, J., Yu, T. H., y Jang, H. R. (2014). The teacher benefits from giving autonomy support during physical education instruction. Journal of Sport \& Exercise Psychology, 36(4), 331-346. http://dx.doi.org/10.1123/jsep.2013-0231

18. Davis, W. (2018). What makes a learning experience intrinsically motivating for american high school language learners? Journal of Pedagogical Research, 2(3), 167-180.

19. Escobar-Pérez, J., y Cuervo-Martínez, A. (2008). Validez de contenido y juicio de expertos: una aproximación a su utilización. Avances en Medición, 6(1), 27-36.

20. Griffin, W. (2016). Perceived autonomy support, intrinsic motivation, and students ratings of instruction. Studies in Educational Evaluation, 51, 116-125. http://doi.org/10.1016/j.stuedduc.2016.10.007

21. Gunuc, S. (2014). The relationships between student engagement and their academic achievement. International Journal on New Trends in Education and Their Implication, 5 (4), 216-231. DOI: 10.5539/ass.v15n11 p1

22. Haerens, L., Aelterman, N., Van der Berghe, L., De Meyer, J., Soenens, B., y Vansteenkiste, M. (2013). Observing physical education teacher's needsupportive interactions in classroom settings. Journal of Sport and Exercise Psychology, 35, 3-17. https://doi.org/10.1123/jsep.35.1.3

23. Haerens, L., Vanstennkiste, M., Aelterman, N., y Van den Berghe, L. (2016). Toward a systematic study of the dark side of the student motivation: Antecedents and consequences of teachers' controlling behaviours. En W. Liu, J. Keng y R. Ryan (Eds.), Building Autonomous Learnes. Perspectives from reseach and practice using self-determination theory (pp. 59-81). Singapore: Springer.

24. Jang, H., Reeve, J., y Deci, E. (2010). Engaging students in learning activities: It is not autonomy support or structure but autonomy support and structure. Journal of Educational Psychology, 102(3), 588-600. http://dx.doi.org/10.1037/a0019682

25. Jang, H., Reeve, J., y Halusic, M. (2016). A new Autonomy-Supportive way of teaching that increases conceptual learning: Teaching in student's preferents ways. The Journal of Experimental Education, 1-16. http://dx.doi.org/10.1080/00220973.2015.1083522

26. Jang, H., Reeve, J., Ryan, R. M., y Kim, A. (2009). Can self-determination theory explain what underlies the productive, satisfying learning experiences of collectivistically-oriented South Korean adolescents? Journal of Educational Psychology, 101, 644-661. https://doi.org/10.1037/a0014241
27. Krijgsman, C., Mainhard, T., van Tarwijk, J., Borghouts, L., Vansteenkiste, M. Aelterman, N., y Haerens, L. (2019): Where to go and how to get there: Goal clarification, process feedback and students' need satisfaction and frustration from lesson to lesson. Learning and Instruction, 61, 1-11. http://doi.org/10.1016/j.learninstruc.2018.12.005

28. Jiang, J., Vauras, M., Volet, S., Salo, A., y Kajamies, A. (2019): Autonomy-supportive and controlling teaching in the classroom: a video-based case study. Education Sciences, 9(3), 229, http://doi.org/10.3390/educsci9030229

29. León, J., Medina-Garrido, E., y Ortega, M. (2018). Teaching quality: high school students' autonomy and competence. Psicothema, 30(2), 218-223. doi:10.7334/psicothema2017.23

30. Meng, H., Wang, J., y Keng, C. (2016). The effectiveness of an autonomy-supportive teaching structure in physical education. RICYDE. Revista Internacional de Ciencias del Deporte, 43(12), 5-28. http://dx.doi.org/10.5232/ricyde2016.04301

31. Moreno-Murcia, J. A. (2016). Supported teaching autonomy support. RICYDE. Revista Internacional de Ciencias del Deporte, 12(43), 2-4. http://dx.doi.org/10.5232/ricyde2015.043ed

32. Moreno-Murcia, J.A., y Sánchez-Latorre, F. (2016). Efectos del soporte de autonomía en clases de educación física. RICYDE. Revista Internacional de Ciencias del Deporte, 43, 79-89. http://dx.doi.org/10.5232/ricyde2016.04305

33. Nuñez, J., y León, J. (2016). The mediating effect of the intrinsic motivation to learn on the relationship between student's autonomy support and vitality and Deep learning. The Spanish Journal of Psychology, 19, e42, 1-18. http://doi.101017/sjp.2016.43

34. Portney, L. G., y Watkins, M. P. (2009). Foundations of Clinical Research: Applications to Practice. 3rd Edition. Philadelphia: F. A. Davis Company.

35. Reeve, J. (2009). Why teachers adopt a controlling motivating style toward students and how they can become more autonomy supportive. Educational Psychologist, 44(3), 159175. doi:10.1080/00461520 903028990.

36. Reeve, J., y Jang, H. (2006). What teachers say and do to support Students' Autonomy during a Learning Activity (2006). Journal of Educational Psychology, 98(1), 209-218. http://dx.doi.org/10.1037/00220663.98.1.209

37. Reeve, J., Jang, H., Carrell, H., Jeon, S., y Barch, J. (2004). Enhancing students' engagement by increasing teachers' autonomy support. Motivation and Emotion, 28(2),

147-169. http://dx.doi.org/10.1023/B:MOEM.0000032312.9549 $9.6 \mathrm{f}$ 


\section{Barrachina-Peris et al.}

38. Reeve, J., \& Tseng, M. (2011). Agency as a fourth aspect of student engagement during learning activities. Contemporary Educational Psychology, 36, 257-267. http://dx.doi.org/10.1016/j.cedpsych.2011.05.002

39. Reeve, J., Vansteenkiste, M., Assor, A., Ahmad, I., Cheon, S. H., Jang, H.;...Wang, C. K. J. (2014). The beliefs that underlie autonomy-supportive and controlling teaching: A multinational investigation. Motivation and Emotion, 38(1), 93-110. http://dx.doi.org/10.1521/jscp.1988.6.3-4.293

40. Reguant-Álvarez, M., y Torrado-Fonseca, M. (2016). El método Delphi. REIRE. Revista d'Innovació $i$ Recerca en Educació, 9(1), 87-102. https://doi.org/10.1344/reire2016.9.1916

41. Rouse, P. C., Duda, J. L., Ntoumanis, N., Jolly, K., y Williams, G. C. (2016). The development and valitacion of the interpersonal support in physical activity consultations observational tool. European Journal of Sport Sciencie, 16(1), 106-114. https://doi.org/10.1080/17461391.2014.987320

42. Ryan, R. M., y Deci, E. L., (2000). Self-determination Theory and the facilitation of the intrinsic motivation, social development, and well-being. American Psychologist, 55(1), 68-78. 10.1037//003-066x.55.1.68

43. Sarrazin, Tessier, Pelletier, Trouilloud, D. y Chanal, J. (2006). The Effects of Teachers' Expectations about Students' Motivation On Teachers AutonomySupportive and Controlling Behaviors. International Journal of Sport and Exercise Psychology, 4, 283-301. https://doi.org/10.1080/1612197X.2006.9671799

44. Schmidt, R. (1997). Managing Delphi surveys using nonparametric statistical techniques. Decision Sciences, 28(3), 763-774. https://doi.org/10.1111/j.1540-5915.1997.tb01330.x

45. Smith, N., Quested, E., Appleton, P., y Duda, J. (2016). A review of observational instruments to assess the motivational environment in sport and physical education settings. International Review of Sport and Exercise Psychology, 9(1), 134-159. https://doi.org/10.1080/1750984X.2015.1132334
46. Skinner, E., Kindermann, T., Connell, J., y Wellborn, J. (2009): Engagement and disaffection as organizational constructs in the dynamics of motivational developement. En K. R. Wenzel y A. Wigfield (Eds.), Educational psychology handbook series. Handbook of motivation at school (pp. 223245). New York, NY, US: Routledge/Taylor \& Francis Group.

47. Smith, N., Tessier, D., Tzioumakis, Y., Quested, E., Appleton, P., Sarrazin, P., Papaioannou, A., y Duda, J. (2015). Developement and validation of the multidimensional motivational climate observation system (MMCOS). Journal of Sport \& Exercise Psychology, 37, 4-22. http://dx.doi.org/10.1123/jsep.2014-0059

48. Soenens, B., y Vansteenkiste, M. (2005). Antecedents and outcomes of self-determination in 3 life domains: the role of parents' and teachers' autonomy support. Journal of Youth and Adolescence, 34(6), 589-604. https://doi.org/10.1007/s10964-005-8948-y

49. Tessier, D., Sarrazin, P., y Ntoumanis, N. (2008). The effects of an experimental programme to support student's autonomy on the overt behaviours of physical education teachers. European Journal of Psychology of Education, 3, 239-253. https://doi.org/10.1007/BF03172998

50. Wallace, T. L., Kelcey, B., y Ruzek, E. A. (2016). What can student perception surveys tell us about teaching? Empirically testing the underlying structure of the tripod student perception survey. American Educational Research Journal, 53(6), 1834-1868. https://doi.org/10.3102/0002831216671864

51. Zang, T., Solmon, M., Kosma, M., Carson, R., y Gu, X (2011): Need suport, need satisfaction, intrinsic motivation, and physical activity participation among middle school students. Journal of Teaching in Physical Education, 30, 51-68. https://doi.org/10.1123/jtpe.30.1.51 